\title{
Development and application of thermoelectric power generation for waste heat recovery
}

\author{
Min $\mathrm{He}^{1, \mathrm{a}}$, Enhua Wang ${ }^{2, \mathrm{~b}^{*}}$, Tong Liü ${ }^{3, \mathrm{c}}$, Fujun Zhang ${ }^{4, \mathrm{~d}}$ and Changlu Zhao ${ }^{5, \mathrm{e}}$ \\ School of Mechanical Engineering, Beijing Institute of Technology, Beijing 100081, China

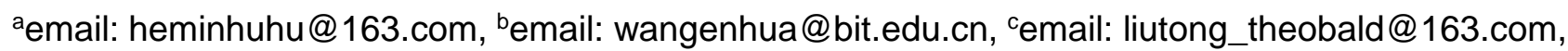 \\ demail: zfj123@bit.edu.cn, eemail: clzhao@bit.edu.cn
}

Keywords: thermoelectric material, power generation, waste heat recovery, application and development

Abstract: Since energy and environmental problems have become severe and urgent which cannot be ignored in the development of modern society, and based on the current situation of energy distribution, this paper gives a review of thermo-electric power generation technology to recover energy. First, a brief introduction of the principle and structure of TEG (Thermoelectric Generator) device is presented. Then, the applications of thermoelectric power generation technology in the recovery of waste heat from internal combustion, industrial plants, daily lives and others are described in detail. The problems existing in actual application of thermoelectric power generation technology are also discussed. All in all, the results of this study can give some directions for further research and development of TEG system.

\section{Introduction}

With the development of modern industrial society, energy shortage and a series of environmental problems caused by energy utilization are not only the critical factors to compete with other countries in China, but also related with the survival and development of human society. Although Chinese energy reservation is not very low around the world, its consumption accounts for $20.3 \%$ of the world's total summation [1], which shows that China has already been the world's largest energy consumer. In addition, China has problems of low energy efficiency per capita, environmental pollution, uneven distribution of energy and so on. In terms of the average recovery of waste heat resources, China is far less than the developed countries. Therefore, waste heat recovery technology is an effective way to make full use of energy and realize the goal of environmental friendly and sustainable development.

Thermoelectric power generation technology is one of the waste heat recovery techniques and one of the three most promising power generation technologies in the 21 st century. It can transform the industrial waste heat, solar energy, geothermal energy, boiler waste heat, exhaust heat of internal combustion engine and other low-grade energy into electric energy whose main theoretical basis is the Seebeck effect. The Seebeck effect which means that heat energy can be directly transformed into electricity by thermoelectric materials, was discovered by the German physicist Seebeck in 1821 [2]. Thermoelectric power generation technology is optimized continuously because of the innovation of materials and equipment structure, its application fields include the aerospace, military, civilian and industrial occasions. TEG device has the advantages of no moving parts, small volume, long service life, no noise at work, environmental friendly and can work under transient temperature, etc. For realizing the objective of the production of clean electricity at present, it has become the most promising technology. As early as in 1963, scientists in the United States had begun to use semiconductor thermoelectric materials for internal combustion engine exhaust waste heat recovery. At that time, because the thermoelectric conversion efficiency is too low and the cost of materials is high, TEGs didn't get a wide range of application.

In recent years, with the breakthrough of material performance and higher energy conversion efficiency of TEG devices, and lower production cost, this technology gets the favor of many researchers again. Thermoelectric power generation technology for thermoelectric conversion 
requirement is not just limited to practicability, reliability and predictability, it but develops gradually towards high performance, low cost, high efficiency [3]. Although thermoelectric power generation technology research has last for two centuries, especially in the recent decade when we advocate energy conservation, emissions reduction, and energy efficient utilization, the study enthusiasm of people is more and more higher, low energy conversion efficiency has been the main obstacle to restrict its large-scale commercial production, especially for low-temperature application. Therefore, it is necessary to summarize the thermoelectric power generation technology in order to find out proper ways to improve the efficiency of thermoelectric conversion.

\section{Principle and structure of thermoelectric power generation technology}

Thermoelectric materials are based on the three basic effects of thermoelectric conversions, namely Seebeck effect, Peltier effect and Thomson effect. The performance parameters of these three effects can be converted to each other, which constitute the index parameters for evaluating thermoelectric devices. The principle of thermoelectric power generation mainly comes from the Seebeck effect. The Seebeck effect is the phenomenon of converting thermal energy into electrical energy. As shown in Fig. 1, when two different conductors form a loop in series, if two connectors 1 and 2 maintain a certain degree of temperature difference, there will be a potential difference in the open position of conductor $b$, that is Seebeck potential.

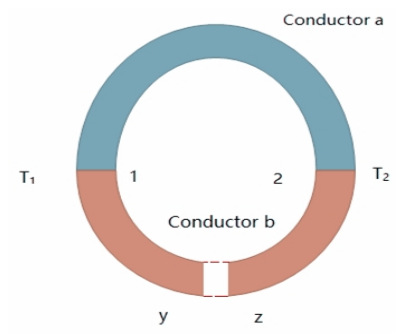

Fig. 1 Thermoelectric effect diagram

Explain from the microscopic point of view, the motion of movable charged particles, which are called carriers, are influenced by the temperature gradients in the conductors. When they gain large kinetic energy from the hot resource, they diffuse toward the cold end, and accumulate. The carriers accumulate on the cold end and further form a self-built electric field. On the other hand, to prevent the carrier from moving toward the cold end and eventually it forms a balance, when the carriers move without direction, the Seebeck potential is formed [4].

The TEG device is generally composed of four modules: a cold module, a thermoelectric module, a hot module, and a clamping equipment [5]. The hot part is made up of a high-temperature heat source such as solar energy, exhaust heat of internal combustion engine. Generally, the hot part and the thermoelectric module are tightly connected through a heat exchanger and a clamping equipment, thereby ensuring a high heat exchange coefficient and a big heat exchange area. The thermoelectric module is composed of a heat-insulating ceramic sheet, the thermoelectric material, and a heat-conducting sheet (generally made of a metal having good thermal conductivity such as copper). And the thermocouples always meet voltage or power requirements through series and/or parallel connection. The cold module and the hot module are opposite to each other to form a temperature difference. Generally, a cooling medium such as air, cooling liquid or heat-conducting oil forms a passage so that the thermoelectric module has sufficient and quick heat dissipation. The clamping equipment ensures that the thermoelectric module contact well with the hot and cold ends so that effective heat conduction and timely heat dissipation can be achieved. In addition, because the power generated by the thermoelectric power generation technology is unstable, the energy storage device may be required to retain the supply and demand of the converted power, the power module may be increased, which is generally composed of current stabilizer and an energy storage system [6]. 


\section{Characteristics}

Waste heat recovery technology can be divided into three categories: low-temperature, medium-temperature and high-temperature according to the temperature range, the last two have been studied from many aspects. The commonly used waste heat recovery technologies include dehumidification, refrigeration, power generation, heat pump technology, heat pipe technology, organic Rankine cycle, and Kalia cycle power generation technology [7,8]. These kinds of recovery technologies can fully utilize energy, recover waste heat reasonably, and reduce environmental pollution to a certain extent, but the overall efficiency is not very high, and there are still other problems, for example the refrigeration recovery technology, we need further improve the refrigeration coefficient and reduce the contamination of the environment; For the heat pipe technology, it exists the problems of compatibility, dew point corrosion, ash and other issues [9].

Compared with waste heat recovery technologies such as organic Rankine cycle [10] and power turbine [11], thermoelectric power generation technology has the advantages of a long power supply life, unlimited application environment and heat sources, high device efficiency, compact structure, small size, low cost and high reliability. In particular, it can use low-grade heat energy such as solar heat and geothermal heat. As we all know, about $70 \%$ of the Earth's surface is composed of oceans, thus the significance and value of ocean thermal power generation technology is extraordinary. Use the temperature difference between warm seawater and deep cold sea water in the sea surface to generate the electricity, the power can be applied to the electricity supply in the surrounding areas of the ocean. At the same time, it is more likely to produce freshwater resources for use for people [12]. At present, with the development of thermoelectric materials, especially the progress of nanomaterials technology, thermoelectric technology has achieved a major breakthrough in thermoelectric conversion efficiency, and it is expected to be commercially applied on a large scale.

\section{Development and application}

Thermoelectric power generation technology is realized in real life by TEG, TEG is initially used in aerospace, then widely to iron and steel industry, cement industry, refinery, and the auto industry and other fields. In addition, TEG can be applied to recover heat from the human body and some of the equipment in life, such as the projector. Loffe proposed to use a merit value $\mathrm{Z}$ to denote the thermoelectric properties of thermoelectric devices.

$$
Z=\frac{S^{2} \sigma}{\kappa} \text {. }
$$

where $S$ is the seebeck coefficient of the material; $\sigma$ is the electric conductivity of the material; $\kappa$ is the thermal conductivity of the material. $S^{2} \sigma$ is called the power factor of the material which is used to measure the electrical transmission performance of the material. The unit of $Z$ is $\mathrm{T}^{-1}, T$ is the average temperature of thermoelectric module, thus $Z T$ is a dimensionaless parameter, which is called the merit value of thermoelectric materials. the larger the value $Z T$, the higher the thermoelectric conversion efficiency. It is generally believed that as long as $Z T$ is greater than 1 or close to 1, TEG will have commercial application value [13]. Therefore, improving $Z T$ value becomes one of the key methods of current development of thermoelectric materials. It is also one of the important factors to reduce the production cost of the thermo-electric power generation technology and improve the efficiency of thermoelectric conversion. The development and application of thermo-electric power generation technology in internal combustion engine are firstly introduced in this paper due to its large scale of study papers on waste heat recovery.

\section{The application of waste heat energy of internal combustion engine}

The thermoelectric power generation technology application for internal combustion engine can be divided into three parts: Thermoelectric materials, generator design and performance analysis, and the thermoelectric power generation technology test research. 
In terms of thermoelectric materials: G. Jeffrey Snyder and Eric S. Toberer summarized the advantages of thermoelectric materials and analyzed the mutual influence characteristics of thermoelectric materials: carrier concentration, effective mass, electronic thermal conductivity, and lattice thermal conductivity. The study which summarized innovative measures of thermoelectric materials shows that thermoelectric materials have been widely studied in the past 50 years and are expected to stimulate a new research boom [14]. Similarly, Jian He and Terry M. Tritt also reviewed some measures to improve the performance of thermoelectric materials. In addition, some of the promising materials were further discussed. It was concluded that the research of thermoelectric materials is an application-driven basic research field. Various subjects such as physics, chemistry, materials science and mechanical engineering disciplines must be linked together. To achieve high material performance, a balanced cooperation must be established to give a full play to multidisciplinary knowledge. At this stage, thermoelectric materials can be classified into low-temperature thermoelectric materials (below $300^{\circ} \mathrm{C}$ ) according to operating temperature, such as $\mathrm{Bi}_{2} \mathrm{Te}_{3}$ and its solid solution alloys, medium-temperature thermoelectric materials $\left(300 \sim 600^{\circ} \mathrm{C}\right)$, such as $\mathrm{PbTe}, \mathrm{SnTe}$, and high-temperature thermoelectric materials $\left(600 \sim 1000{ }^{\circ} \mathrm{C}\right)$, such as SiGe, $\mathrm{MnTe}$, $\mathrm{SiRe}_{2}$, etc. [15]. At present, the recreation for high-quality thermoelectric materials is the research direction of thermoelectric materials. The main research aspects include: researches on innovate thermoelectric materials, researches on creative micro structures and researches on preparation method of new processes.

As for the TEG design and performance analysis: different shapes of the exhaust pipe will affect the layout of the thermoelectric device, and then influence the power generation performance. Tae Young Kim from the Korea University of Science and Technology, took a turbocharged six-cylinder diesel engine as the study object and recovered the rectangular exhaust pipe's waste energy. The study applied the layout of up and down $4 \times 5 \times 2$ of a total 40 pieces of thermoelectric modules, and did real-stuff experiments under different conditions. The results showed that the output power increases with the increasing of load and speed, and the largest thermoelectric conversion efficiency is $2.8 \%$ [16]. Liu Wei from Tianjin University used CFD software to carry out a thermodynamic analysis on the exhaust gas of a large-displacement diesel engine with a cylindrical exhaust pipe. The results showed that the exhaust back pressure at the front and rear ends of the heat exchanger and the size of the diversion device would affect the conversion between thermal energy and electrical energy. Under the condition of optimal design of the flow guiding device, the net output power of the system can be increased up to $75.2 \%$ [17]. Wang et al. designed a metal foam-filled TEG device and studied the change of power generation performance of the TEG system by changing the pores density of the metal foam. At the same time, the paper explained that the inlet temperature at the hot end, the flow rate at the cold end, and the thermoelectric module connection method have important impacts on the power efficiency, heat transfer effect, and waste heat recovery rate [18]. From the results of the research on thermoelectric device design and performance analysis, it can be concluded that the arrangements of the hot and cold modules and the thermoelectric module of the TEG device have an important influence on the performance of the entire generator. In addition, the small changes in thermoelectric devices will affect the Seebeck coefficient, electrical conductivity, and thermal conductivity correspondingly and largely. Whereas, The variation tendency of these three parameters is often inconsistent, thus the impact on the merit value $Z T$ still could be unknown

Experimental investigations for thermoelectric power generation: During the theoretical research process of the TEG device, the heat transfer and dissipation and the structural design of the thermoelectric material are very important. However, the matching design of the thermoelectric generator and the corresponding research object such as internal combustion engine must also be considered. A. Massaguer et al. took the light vehicle as the subject, mainly tested and analyzed the power generation performance of thermoelectric devices under different steady-state conditions and transient New European Driving Cycle (NEDC) conditions. The results showed that in practical applications, the thermodynamics inertia and pressure drop need to be paid attention to for thermoelectric devices, and compared with the transient running conditions, the power generated by 
the working process of steady working conditions is larger [19]. Part of the research results are as follows:

Table 1 Literatures on the experimental study of TEGs

\begin{tabular}{|c|c|c|c|}
\hline $\begin{array}{l}\text { Research } \\
\text { organization }\end{array}$ & Heat resource & Materials & Results \\
\hline $\begin{array}{l}\text { Institute of } \\
\text { Machinery and } \\
\text { Materials in } \\
\text { Korea }^{[20,21]}\end{array}$ & exhaust & $\mathrm{Bi}_{2} \mathrm{Te}_{3}$ & $\begin{array}{l}\text { With the increasing of engine speed or load, the } \\
\text { output power of the thermoelectric generator } \\
\text { increases, and the thermoelectric efficiency } \\
\text { measured is } 1.3 \sim 2.8 \% \text {. }\end{array}$ \\
\hline $\begin{array}{l}\text { Loughborough } \\
\text { University, } \\
\text { Britain }^{[22]}\end{array}$ & exhaust & $\begin{array}{l}\text { GM250-127-28-1 } \\
0\end{array}$ & $\begin{array}{l}\text { Through the modeling analysis, the main structural } \\
\text { parameters affecting the TEG are determined and the } \\
\text { output performance under transient condition is } \\
\text { tested by experiment. The output efficiency of the } \\
\text { optimized TEG can be increased by } 25 \% \text {. }\end{array}$ \\
\hline $\begin{array}{l}\text { University of } \\
\text { konmhollier, } \\
\text { Turkey }\end{array}$ & exhaust & $\mathrm{Bi}_{2} \mathrm{Te}_{3}$ & $\begin{array}{l}\text { The test on the Fiat P8602 diesel engine shows that } \\
\text { when the engine speed is } 3500 \mathrm{r} / \mathrm{min} \text { and the output } \\
\text { torque is } 100 \mathrm{Nm} \text {, the TEG power is } 156 \mathrm{~W} \text {. }\end{array}$ \\
\hline $\begin{array}{l}\text { France's New } \\
\text { Energy and } \\
\text { Atomic Energy } \\
\text { Commission }{ }^{[24]}\end{array}$ & exhaust & $\begin{array}{l}\mathrm{Bi}_{2} \mathrm{Te}_{3} \text { and } \\
\text { silicon-germaniu } \\
\text { m alloys }\end{array}$ & $\begin{array}{l}\text { Experimental results show that the output power of } \\
\text { bismuth telluride are } 70 \% \text { higher than that of silicon } \\
\text { germanium alloy, but when the exhaust temperature } \\
\text { higher than } 300^{\circ} \mathrm{C} \text {, bismuth telluride thermoelectric } \\
\text { materials' output performance degenerates rapidly. }\end{array}$ \\
\hline $\begin{array}{l}\text { Dalian Maritime } \\
\text { University }\end{array}$ & $\begin{array}{l}\text { Diesel engine flue } \\
\text { gas }\end{array}$ & $\begin{array}{l}\text { Different } \\
\text { temperatures' } \\
\text { semiconductor } \\
\text { materials. }\end{array}$ & $\begin{array}{l}\text { Based on various TEGs devices, the output power is } \\
8.45 \mathrm{kw} / \mathrm{m}, 3.55 \mathrm{kw} / \mathrm{m} \text { and } 9.45 \mathrm{kw} / \mathrm{m} \text { respectively. } \\
\text { Thermoelectric efficiency is } 4.01 \%, 4.06 \% \text { and } \\
4.36 \% \text { respectively. }\end{array}$ \\
\hline $\begin{array}{l}\text { China } \\
\text { Shipbuilding } \\
\text { Industry } \\
\text { Corporation }^{[26]}\end{array}$ & exhaust & TEP1 & $\begin{array}{l}\text { Using the TEG test device with the heat conducting } \\
\text { oil circulation for exhaust energy, the power } \\
\text { generation efficiency is } 1.31 \sim 2.38 \% \text {. }\end{array}$ \\
\hline $\begin{array}{l}\text { East China } \\
\text { University Of } \\
\text { Science and } \\
\text { Technology } \\
\end{array}$ & $\begin{array}{l}\text { Medium \& low } \\
\text { temperature gases } \\
\text { and high } \\
\text { temperature liquids }\end{array}$ & $\begin{array}{l}\text { Carbon nanotubes } \\
\text { doped with } \\
\text { bismuth telluride }\end{array}$ & $\begin{array}{l}\text { Through the design of thermoelectric material and } \\
\text { heat exchanger structure, the heat transfer efficiency } \\
\text { increases to } 86.98 \% \text {, the maximum open-circuit } \\
\text { voltage reaches to } 1.95 \mathrm{~V} \text {, and the power generation } \\
\text { efficiency reaches to } 2.20 \% \text {. }\end{array}$ \\
\hline $\begin{array}{l}\text { Dalian Maritime } \\
\text { University[27] }\end{array}$ & jacket water & $\mathrm{Bi}_{2} \mathrm{Te}_{3}$ & $\begin{array}{l}\text { The experimental device is set up, and the influence } \\
\text { factors of contact heat resistance, contact pressure, } \\
\text { hot end temperature, load resistance and power strip } \\
\text { connection are carried out. }\end{array}$ \\
\hline
\end{tabular}

\section{Other applications of energy recovery}

thermo-electric power generation technology is used not only in heat recovery of internal combustion engine but also for geothermal energy, living heat, industrial waste heat and others.

Chen Haiping from North China Electric Power University combined thermo-electric power generation technology with a set of known geothermal data. The experiment focused on the output power change with the temperature of hot resource of a single semiconductor thermocouple when the load is constant, and the semiconductor one's matching load while the output power is maximum. The paper also took geothermal parameters of Yangbajing, Tibet to verify the practicality and rationality of Geothermal -TEG system [28]. Nowadays, the development and application of renewable energy has always been a focus for researchers, especially for clean solar energy. Liang Qiuyan combined solar collectors and semiconductor thermoelectric technologies, then designed a related device, and furtherly studied the thermoelectric coupling performance and its key techniques, which showed that the maximum thermoelectric conversion efficiency is $5.4 \%$, and the maximum efficiency of the device is $4.1 \%$. The electric energy within 10 days of the device can meet the electricity demand in a small nursery greenhouse [29]. 
The large amount of radiant waste heat generated in the industrial production process can also be recycled through Thermo-electric power generation, which not only reduces energy waste, but also alleviates environmental pollution and safety issues. Fan Yudong from Anhui University of Technology designed a set of TEG device for low-temperature heat radiation energy recovery. The paper studied the influence of cooling conditions, heat radiation area, and heat radiation distance and others on the power generation performance of the thermoelectric device. The results show that the smaller the heat radiation distance is, the greater the power generated, and the thermoelectric power is bigger because of water cooling system [30].

\section{Conclusions}

Thermoelectric power generation technology can improve the utilization of low-grade thermal energy and reduce environmental pollution. In this study, the principle and structure of the thermoelectric power generation device is introduced briefly at first. The application of thermoelectric power generation technology in the recovery of internal combustion waste heat and other industrial heat sources are presented in detail.

Since the 1950s, every rising of the conversion efficiency of TEG brings the research and development upsurge. According to the report, a lot of thermoelectric materials merit ZT has reached 1.5 in theory and the efficiency has been up to $20 \%$. However, the actual application of the transient condition will reduce the conversion efficiency, it is generally less than $4 \%$ for a vehicle-TEG device. On the other hand, the efficiency of a TEG for geothermal sources is no more than $3 \%$ currently.

At present, the thermo-electric power generation technology researches mainly focus on the thermoelectric materials development and TEG device structure optimization. Meanwhile, thermal power generation device with good heat transfer and heat dissipation performance should be designed to improve the efficiency of conversion. Although TEG has many advantages, low energy conversion efficiency is still the biggest shortcoming of the technology. There are still many problems to be solved. Nevertheless, it is a potential technology for the future sustainable society.

\section{Acknowledgements}

This work was financially supported by the Research Startup Program of Beijing Institute of Technology.

\section{References}

[1] Wang Tongcai. Low and medium temperature waste heat recovery using thermoelectric power generation[D]. East China University of Science and Technology, 2017.

[2] Chen lingen, Meng Fankai, Geyanlin et al. Research on thermodynamic research of semiconductor thermoelectric devices[J]. Journal of Mechanical Engineering, 2013,49(24):144-154.

[3] He J, Tritt T M. Advances in thermoelectric materials research: Looking back and moving forward[Z]. 2017.

[4] Gao min, Zhang Jingshao, D. M. Rown. Thermoelectric conversion and its application[M]. Beijing: Weapons Industry Press, 1996.

[5] Yuan Xiaohong. Automotive engine exhaust gas residual heat thermoelectric power generator for the thermoelectric conversion technology research[D]. Wuhan University of Technology, 2012.

[6] Ji Baoning. Temperature difference energy transformation and energy power generation system matching technology research[D]. Harbin Industrial University, 2017.

[7] Peng Hanming, Yang Minlin, Jiang Runhua, Qin Guanfeng. Low temperature waste heat recovery technology in the distributed energy systems[J]. Energy Saving, 2011, (3): 4-8.

[8] Feng Huisheng, Xu Feifei, Liu Yefeng, Dan Chun. Industrial process waste heat recycling technology research progress[J]. Journal of Chemical Industry and Engineering, 2012, (01): 57-64. 
[9] Xu Weiwei, Tang Xiaodong, et al. Review on low temperature waste heat recovery upgrade technology[J]. Journal of Chemical Industry in Guangzhou, 2011, (23): 34-36.

[10] Wang E, Yu Z, Zhang H, Yang F. A regenerative supercritical-subcritical dual-loop organic Rankine cycle system for energy recovery from the waste heat of internal combustion engines[J]. Applied Energy, 2017, 190: 574-590.

[11] Katsanos CO, Hountalas DT, Zannis TC. Simulation of a heavy-duty diesel engine with electrical turbocompounding system using operating charts for turbocharger components and power turbine[J]. Energy Conversion and Management, 2013,76: 712-724.

[12] Yan liqiang, Cheng Jiang, Liu Maoyuan. Introduction to temperature difference power generation[J]. Journal of Solar Energy, 2015. (01): 11-15.

[13] Zhao Kunyu, Ge Zhenhua, Li Zhidong. Introduction to new thermoelectric materials[M]. Beijing: Science Press, 2018:1-13.

[14] Snyder G Jeffrey, Toberer Eric S. Complex thermoelectric materials[J]. Nature Materials, 2008, $7(2)$.

[15] Xu lizhen, li yan, Yang zhi, et al. Experimental study on the generation of automobile exhaust temperature difference [J]. Journal of Tsinghua University, 2010,50 (2): 287-289.

[16] Tae Young Kim, Assmelash A, Negash, Gyubaek Cho. Waste heat recovery of a diesel engine using a thermoelectric generator equipped with customized thermoelectric modules[J]. Energy Conversion and Management, 2016,124.

[17] Liu Yi. Study on the thermal power generation system of exhaust waste heat recovery of diesel engine with large temperature difference [D]. Tianjin University, 2016.

[18] Wang T, Luan W, Liu T, et al. Performance enhancement of thermoelectric waste heat recovery system by using metal foam inserts[J]. Energy Conversion and Management. 2016, 124:13-19.

[19] Massaguer A, Massaguer E, Comamala M, et al. Transient behavior under a normalized driving cycle of an automotive thermoelectric generator[J]. Applied Energy. 2017, 206: 1282-1296.

[ 20] Kim TY, Negash AA, Cho G. Waste heat recovery of a diesel engine using a thermoelectric generator equipped with customized thermoelectric modules[J]. Energy Conversion and Management, 2016, 124: 280-286.

[21] Kim TY, Kwak J, Kim B. Energy harvesting performance of hexagonal shaped thermoelectric generator for passenger vehicle applications: An experimental approach[J]. Energy Conversion and Management, 2018, 160: 14-21.

[22] Lan S, Yang Z, Chen R, et al. A dynamic model for thermoelectric generator applied to vehicle waste heat recovery[J]. Applied Energy, 2018, 210: 327-338.

[23] Temizer I, Ilkilic C. The performance and analysis of the thermoelectric generator system used in diesel engines[J]. Renewable and Sustainable Energy Reviews, 2016, 63: 141-151.

[24] Nour Eddine A, Chalet D, Faure X, et al. Optimization and characterization of a thermoelectric generator prototype for marine engine application[J]. Energy, 2018, 143: 682-695.

[25] Zheng Zisheng. Experimental study on the utilization of residual heat in ships based on semiconductor temperature difference[D]. Dalian Maritime University, 2017.

[26] Zheng Xiyang, Wang Zhigang, Zhong Qingyuan. Research on the thermal power generation technology of exhaust heat of Marine diesel engines [J]. Jiangsu Ship, 2016, 33(6): 15-17.

[27] Wang Hongming. Design and experimental study of residual thermal power generation devices in ships [D]. Dalian Maritime University, 2016.

[28] Chen Haiping, Yu Xinwei Shi Zhiyun, Wang Zhongping, Wu Wenhao. Geothermal temperature difference power generation system of experiment research and economical analysis[J]. Electric Power Science and Engineering, 2013, (02): 63-63.

[29] Liang Qiuyan. Concentrated solar thermoelectric performance of the key techniques and thermoelectric generator mechanism research[D]. The Northeast Agricultural University, 2016.

[30] Fan Yudong. Industrial radiation directly generating research [D]. Anhui University of Technology, 2017. 